\title{
Observations on the Consortium Method of Infection Control
}

The consortium approach to hospital infection control has lately become a subject of interest. In the past year, I have received a number of queries about methodological aspects, such as: What are specific services a consortium provides? What are important day-to-day problems? How are hospital payments determined? What areas are likely to yield good results from cost-containment efforts? What is the future of consortia in light of current hospital constraints? I shall try to respond by offering general comments from a vantage of close to 15 years of experience. Specific details of structure, function and efficacy of one consortium are presented elsewhere. ${ }^{1,2}$

The concept of an infection control consortium is simple-several hospitals do for all what none can do alone. The hospital group acquires services of consultants, teachers for infection control practitioners (ICPs) and standardization of policies that incorporate local concerns. To make the organization function in a meaningful way, participating hospitals must be willing to share infection occurrence data, as well as educational and surveillance costs, and have a genuine interest in improving standards of infection control. In turn, the managers of the organization must be effective in their roles, mindful of the need to protect confidentiality of data sources and provide objectivity in analyses. A consortium is welladapted to serve community hospitals that do not have

From the South Florida Hospital Consortium for Infection Control, Miami, Florida.

Address reprint requests to $N$. Joel Ehrenkranz, MD, Director, South Florida Hospital Consortium for Infection Control, $1295 \mathrm{NW}$ 14th Street, Suite M, Miami, FL 33125. ready access to infection control epidemiologists (not to be confused with infectious disease clinicians).

Consortium services may provide:

- Direct participation of consultants in a variety of hospital activities relating to infection control; namely, various committee meetings, on-site inspections, evaluation of planned construction or remodeling, outbreak work-ups, antibiotic audits, workshops for employees and medical staff, discussions with risk managers, testimony as expert witness during litigation, and opinions at news conferences;

- Ongoing education for ICPs to improve their skills and worth as hospital resources;

- Formulation of policies modified by local considerations when necessary;

- Efforts at cost-containment;

- Surveillance and comparison of endemic nosocomial infection frequencies to determine expected infection rates. The objectives are to identify the best level of control of infection as well as hyperendemic excesses, and to recognize variations in infection occurrences because of seasonal, periodic, and secular trends;

- Efforts to influence legislation related to infection control;

- Product evaluation; and

- Research in infection control.

A consortium will inevitably require considerable expenditure of time by a number of individuals as well as expenditure of resources to develop and nurture the group. It is essential to recruit member hospitals and people (consultants and educators), assess common and individual needs of members, establish methodology, col- 
lect and analyze data, investigate problems, deal with regulators and public health authorities, and, on occasion, respond to hospital critics. For the director, some managerial experience is invaluable.

To avoid unnecessary efforts and repetitive activities, it is useful to explore the different methods by which change can be brought about in the various constituents. It may not be simple to induce independent hospitals to act in a cohesive way, despite sound and persuasive arguments. In dealing with outbreaks, personnel of the consortium will find it productive in the long term to be able to cope with frustration in order to achieve goals. In the event of success, the role of the Hawthorne effect should be acknowledged. Along the way there may be spirited discussions with surgeons about antibiotic restrictions, internists about placement of patients, intravenous and respiratory therapists about the uses of various filters, pharmacists about environmental cultures, representatives of industry about choices of products, and junior hospital administrators and department managers about perceived territorial infringements. Occasional confrontations can be unpleasant. Nonetheless, it is crucial (albeit difficult at times) to maintain focus solely on the problem.

Hospital payment should be based on the risks of nosocomial infection implicit in the complexity of patient services and the organizational efforts necessary for infection control. Total payments should be adequate to compensate consortium personnel proportionate to their efforts and to support the basic organizational structure. Research undertakings should be borne by separate research funding; however, development of programs that ultimately provide regular services may be supported by general revenues. At the outset, it is helpful to secure "start up" money-perhaps the equivalent of 1 to 2 years of estimated financial needs-so that problems of undercapitalization can be minimized. There should be a contract to define the responsibilities of the consortium and each member hospital; issues of confidentiality, liability, and nonperformance should be addressed.

Cost-containment may be productively explored along the following lines: 1) reduction of nosocomial infectious occurrences, which result in costly patient services, prolonged hospital stay, or legal exposure; 2) recognition of hidden infection control costs in planning new programs (eg, in hospitals with oncology centers there may be more critical care outbreaks than in hospitals without such programs);2 3) elimination of unnecessary infection control practices; and 4) truncation of useful but costly practices. Results of cost-containment efforts at one consortium have been reported elsewhere. ${ }^{1}$ Suffice it to say that it is relatively easy to demonstrate possibilities for considerable savings among unsophisticated hospitals early in their membership, but much more difficult to show similar opportunities among long-term members employing a seasoned infection control staff.

For continued success, it is essential to develop broad group representation for decision-making, ie, involvement of member hospital ICPs, infection committee chairpersons, administrative and laboratory personnel, and others who may be interested and are capable of working for a common goal. For consultants, clinical competence is valuable in establishing credibility with medical and nursing staffs. However, for consulting physicians the potential exists for a conflict of interest if they derive considerable income from clinical activities at a single hospital. To the extent possible, individual hospital payments should not be so large as to create undue dependence on a single institution. It is important to voice "loyal opposition" when necessary.

What is the future? A number of community hospitals currently lack medical staff with expertise in infection control. If Petersdorf $\mathrm{f}^{3}$ and Ervin ${ }^{4}$ are correct in predicting a decrease in numbers of physicians entering private infectious disease practice, the situation can only deteriorate. The majority of infectious disease physicians whom I have met in a private practice setting possess but limited knowledge or interest in infection control theory. In this regard the findings of a 1980 survey are noteworthy; only $54 \%$ of 121 adult infectious disease training programs reported the presence of a faculty member with a major interest in hospital epidemiology. ${ }^{5}$ Should pure infectious disease practice revert primarily to a university activity, infection control in private hospitals will surely suffer and, pari passu, important measures that bear on the quality of patient care. The consortium approach is a method whereby individuals who are involved in community hospital infection control can be both effective and efficient.

\section{REFERENCES}

1. Ehrenkranz $\mathrm{NJ}$ : The South Florida Hospital Consortium for Infection Control: Structure and function. Am J Infect Control (in press).

2. Ehrenkranz NJ: The efficacy of a Florida hospital consortium for infection control: 1975-1982. Infect Control 1986; 7:321-326.

3. Petersdorf RG: Whither infectious diseases? Memories, manpower and money. Infect Dis 1986; 153:189-195.

4. Ervin FR: The bell tolls for the infectious disease clinician. J Infect Dis 1986; 153:183-185.

5. Shands SW, Wenzel RP, Wolff SM, et al: Hospital epidemiology and infection control: The changing nature of the specialist in infectious diseases. J Infect Dis $1981 ; 144: 609-613$. 